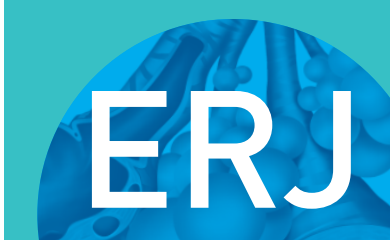

open research

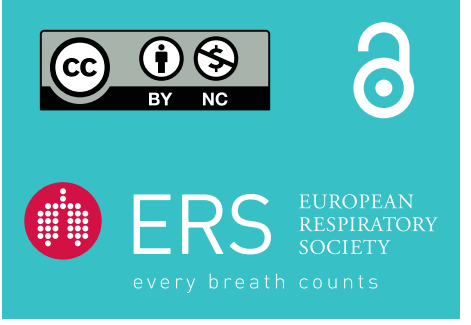

\section{Nicotine treatment and smoking cessation in the era of COVID-19 pandemic: an interesting alliance}

To the Editor:

Since the declaration of the coronavirus disease 2019 (COVID-19) outbreak as a pandemic, the worldwide medical community has been racing against time to identify effective therapeutic agents to constrain the disease and reduce the effects of severe acute respiratory syndrome-coronavirus-2 (SARS-CoV-2) infection. Recently, the nicotinic hypothesis has been proposed; according to this, the acetylcholine receptor (nAChR) might play a key role in the pathophysiology of COVID-19 and, thus, should be a potential therapeutic target to reduce COVID-19 burden and its complications [1]. Since nicotine replacement therapy has long been used to treat tobacco dependence, this is an interesting hypothesis with important implications.

The infectivity and systemic manifestations of SARS-CoV-2 seem to be, at least partially, modulated via the renin-angiotensin-aldosterone system (RAAS), which is an elegant cascade of vasoactive peptides that orchestrate key processes in human physiology [2]. SARS-CoV-2 invades the human cells after binding to its functional receptor, that is angiotensin-converting enzyme 2 (ACE2), which is found in several organs, including the heart and the kidneys, but it dominates on lung alveolar epithelial cells [3]. This virus binding to ACE2 potentially alters the function of the latter, resulting in massive loss of ACE2 function, enhancing the pathogenetic process of cardiorespiratory failure (figure 1) [3]. The loss of ACE2 has proved to induce the production of reactive oxygen species in animal models [5], while it may also have deleterious effects on cardiac and lung structures and function, due to the unopposed angiotensin II accumulation $[2,6]$.

Nicotine is a cholinergic agent that modulates RAAS [1, 7]. However, published data regarding the exact effect of nicotine on ACE2, in relation to COVID-19 infection, are somehow contradictory. There are studies which indicate that nicotine downregulates ACE2 levels, thereby decreasing the number of potential virus "entry-points" into the cells, which could reduce its infectivity [1]. Contrary to that, other data suggest that nicotine might upregulate ACE2 levels. Recently, Russo et al. [8] indicated in vitro induction of ACE2 in human lung alveolar epithelial cells, which were continuously treated with nicotine; this upregulation was conducted only through the nicotinic acetylcholine receptor $\alpha 7$ subunit $(\alpha 7$-nAChRs) signalling pathway (figure 1). Although ACE2 upregulation may promote cellular uptake of SARS-CoV-2 [8], other authors suggest that it may also be a defensive mechanism to counteract the deleterious effects of angiotensin II, resulting in less organ damage and reduced disease severity [7]. Since there is as yet no definite evidence on whether nicotine administration may affect COVID-19 progression through the RAAS, this is a hypothesis that warrants further investigation.

Another interesting hypothesis is that nicotine might have an impact on the course of COVID-19 through its properties as an anti-inflammatory agent and a neurotransmitter. Nicotine is an important inhibitor of several pro-inflammatory cytokines, such as interleukin (IL)-1, IL-6 and tumour necrosis factor, acting through the cholinergic anti-inflammatory pathway via the $\alpha 7-n A C h R s$ receptor of the macrophages (figure 1) [7]. Since cytokine storm appears to be the hallmark in severe COVID-19 cases [7, 9], one could

@ERSpublications

In the era of this pandemic, the role of medicinal nicotine in the prevention and treatment of \#COVID19 disease should be evaluated in placebo-controlled trials, while smoking cessation should be further promoted as a general public health measure https://bit.ly/3fpsBdq

Cite this article as: Boutou $\mathrm{AK}$, Pitsiou G, Kontakiotis $\mathrm{T}$, et al. Nicotine treatment and smoking cessation in the era of COVID-19 pandemic: an interesting alliance. ERJ Open Res 2020; 6: 003062020 [https://doi.org/10.1183/23120541.00306-2020].

Copyright $\odot$ ERS 2020. This article is open access and distributed under the terms of the Creative Commons Attribution NonCommercial Licence 4.0. 


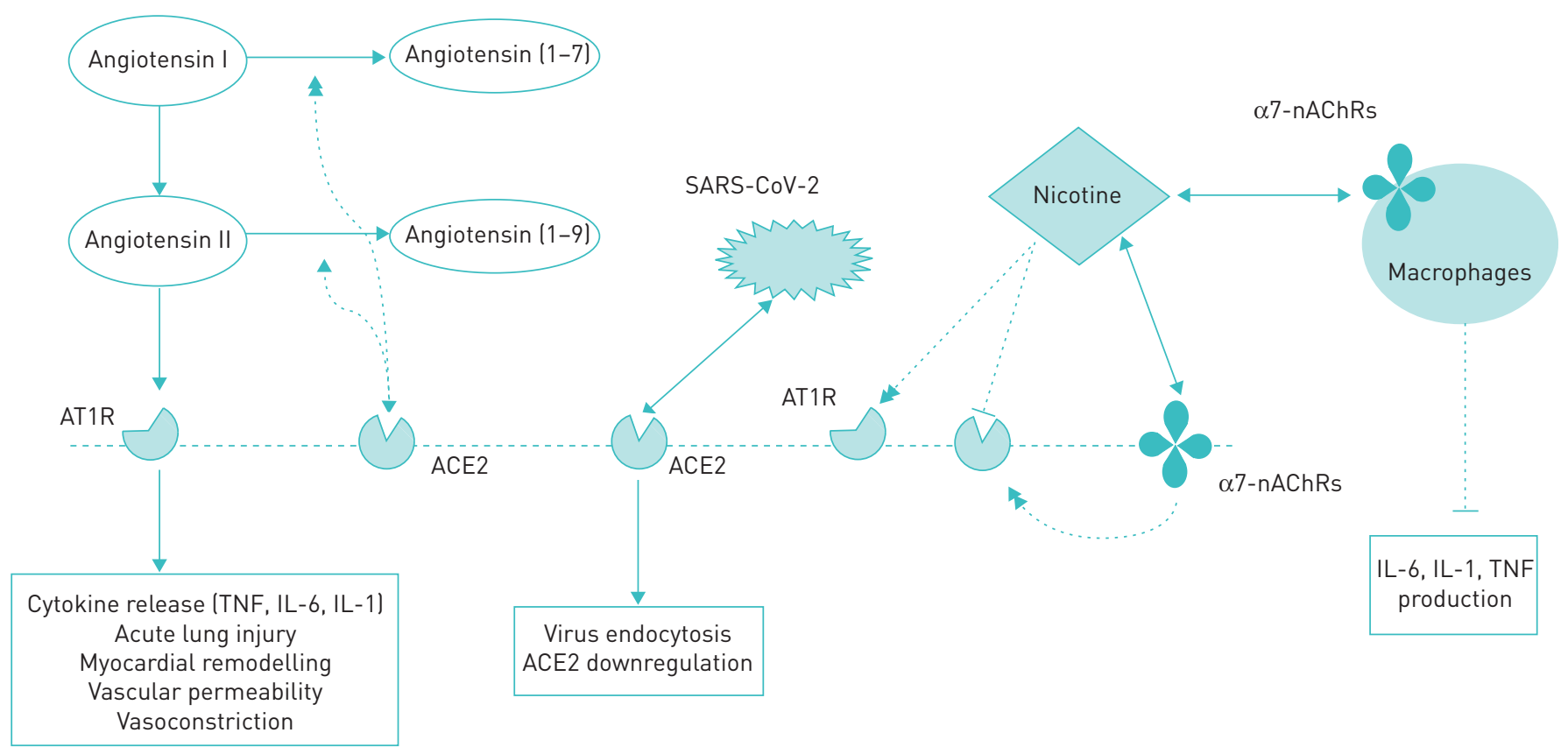

FIGURE 1 The complex interaction between severe acute respiratory syndrome-coronavirus-2 (SARS-CoV-2), nicotine and the renin-angiotensin-aldosterone system. SARS-CoV-2 binds to the its functional receptor on alveolar epithelial cells, that is angiotensin-converting enzyme (ACE)2. This binding results in viral endocytosis and replication, along with ACE2 downregulation. Nicotine might interact with RAAS in multiple ways: 1) by upregulating angiotensin II type I receptor, and thus by inducing angiotensin II deleterious effects; and 2) by downregulating ACE2, and thus reducing the number of potential virus entries or by upregulating ACE2, via nicotinic acetylcholine receptor $\alpha 7$ subunit $(\alpha 7-n A C h R)$. The latter signalling pathway may also result in the downregulation of cytokine production by the macrophages [2, 4]. AT1R: angiotensin II type I receptor; TNF: tumour necrosis factor; IL: interleukin.

hypothesise that exogenous nicotine administration might enhance the cholinergic anti-inflammatory pathway and contribute to maintaining a balanced immune response against the virus infection [7]. However, other published data suggest that nicotine exposure promotes respiratory viral infections, such as influenza, through the impaired cytokine signalling and suppressed leukocyte migration [10]; thus, whether the immunomodulatory effect of nicotine regarding COVID-19 infection might be favourable or not needs to be further investigated. Moreover, neuroinvasion is a common feature of SARS-CoV-2 infection, while anosmia and ageusia are frequent manifestations among COVID-19 patients. Similarly, in patients with Parkinson's disease, oflaction is reduced early in the course of the disease, as a sign of abnormal cholinergic transmission [11], while data from animal models of Parkinson's disease suggest that nicotine may improve oflactory impairment [12]. Again, the potential efficacy of nicotine supplementation for COVID-19 patients with anosmia, needs to be further tested.

Under this scope, is there any potential role of smoking in the era of the pandemic? The answer given by all health providers has to be unanimous and definite: no! Apart from nicotine, tobacco preparations contain more than 5000 chemical compounds, hundreds of which are known carcinogens or are associated with several cardiovascular or respiratory disorders [13]; the presence of these comorbidities is a major risk factor both for infection by SARS-CoV-2 and the development of critical disease, among infected patients $[14,15]$. With regards to COPD, recently published data indicate that it is andependent risk factor for severe COVID-19 disease [16] and for intensive care unit admission [17] due to the SARS-CoV-2 infection. Smoking exposure is associated with a diminished, early epithelial-initiated antiviral response to other respiratory viruses, such as rhinovirus, an effect which could contribute to the increased susceptibility of subjects to prolonged respiratory viral infections [18]. Smoking is also known to cause lung damage through the activation of inflammatory cytokines, and to affect the function of circulating immune cells [11], actions that may also contribute to COVID-19 disease outcome. In agreement to these, current smoking was recently identified as an independent predictor of severe complications and increased mortality among COVID-19 patients [19, 20].

In the era of the pandemic, the two approaches may both prove to be beneficial. Medicinal nicotine should be rapidly examined in placebo-controlled trials for a potential role in the prevention and treatment of COVID-19 disease, and plans for such a trial were recently announced by French researchers [1]. Nicotine preparations are approved worldwide, have been used for decades and have an appealing safety profile, 
even when they are used in populations with several comorbidities as they have few contraindications, are not expensive and are easily available [1]. Moreover, smoking cessation should be further motivated, as a general public health measure. There is a risk, though: nicotine preparations to be massively bought over-the-counter without evidence of efficacy and smoking cessation to be abandoned, although it is the single most effective intervention to increase life expectancy in the general population. Thus, strong scientific evidence, adequate health system awareness and organisation, and reliable media campaigns are all crucial in order for this alliance to work.

\section{Afroditi K. Boutou $\oplus^{1}$, Georgia Pitsiou ${ }^{2}$, Theodore Kontakiotis ${ }^{3}$ and Ioannis Kioumis ${ }^{2}$}

${ }^{1}$ Dept of Respiratory Medicine, G. Papanikolaou Hospital, Thessaloniki, Greece. ${ }^{2}$ Dept of Respiratory Failure, Aristotle University of Thessaloniki, Thessaloniki, Greece. ${ }^{3}$ Dept of Respiratory Medicine, Aristotle University of Thessaloniki, Thessaloniki, Greece.

Correspondence: Afroditi K. Boutou, Dept of Respiratory Medicine, G. Papanikolaou Hospital, 57010, Exohi, Thessaloniki, Greece. E-mail: afboutou@yahoo.com

Received: 15 May 2020 | Accepted after revision: 1 July 2020

Conflict of interest: None declared.

\section{References}

1 Tindle HA, Newhouse PA, Freiberg MS. Beyond Smoking Cessation: Investigating Medicinal Nicotine to Prevent and Treat COVID-19. Nicotine Tob Res 2020: ntaa077.

2 Vaduganathan $\mathrm{M}$, Vardeny $\mathrm{O}$, Michel $\mathrm{T}$, et al. Renin-angiotensin-aldosterone system inhibitors in patients with Covid-19. N Engl J Med 2020; 382: 1653-1659.

3 Alifano M, Alifano P, Forgez P, et al. Renin-angiotensin system at the heart of COVID-19 pandemic. Biochimie 2020; 174: 30-33.

4 Fudim M, Qadri YJ, Ghadimi K, et al. Implications for neuromodulation therapy to control inflammation and related organ dysfunction in COVID-19. J Cardiovasc Transl Res 2020; .

5 Patel VB, Zhong J-C, Grant MB, et al. Role of the ACE2/Angiotensin 1-7 axis of the renin-angiotensin system in heart failure. Circ Res 2016; 118: 1313-1326.

6 Gheblawi M, Wang K, Viveiros A, et al. Angiotensin-converting enzyme 2: SARS-CoV-2 receptor and regulator of the renin-angiotensin system. Circ Res 2020; 126: 1456-1474.

7 Farsalinos K, Niaura R, Le Houezec J, et al. Nicotine and SARS-CoV-2: COVID-19 may be a disease of the nicotinic cholinergic system. Toxicol Rep 2020; 7: 658-663.

8 Russo P, Bonassi S, Giacconi R, et al. COVID-19 and smoking: is nicotine the hidden link? Eur Respir J 2020; 55: 2001116.

9 Mehta P, McAuley DF, Brown M, et al. COVID-19: consider cytokine storm syndromes and immunosuppression. Lancet 2020; 395: 1033-1034.

10 Razani-Boroujerdi S, Singh SP, Knall C, et al. Chronic nicotine inhibits inflammation and promotes influenza infection. Cell Immunol 2004; 230: 1-9.

11 Bohnen NI, Müller MLTM, Kotagal V, et al. Olfactory dysfunction, central cholinergic integrity and cognitive impairment in Parkinson's disease. Brain J Neurol 2010; 133: 1747-1754.

12 Yang J, Lv D-J, Li L-X, et al. Nicotine improved the olfactory impairment in MPTP-induced mouse model of Parkinson's disease. Neurotoxicology 2019; 73: 175-182.

13 Olds JL, Kabbani N. Is nicotine exposure linked to cardiopulmonary vulnerability to COVID-19 in the general population? FEBS J 2020; .

14 Liang W, Liang $\mathrm{H}, \mathrm{Ou} \mathrm{L}$, et al. Development and validation of a clinical risk score to predict the occurrence of critical illness in hospitalized patients with COVID-19. JAMA Intern Med 2020; .

15 Lian J, Jin X, Hao S, et al. Epidemiological, clinical, and virological characteristics of 465 hospitalized cases of coronavirus disease 2019 (COVID-19) from Zhejiang province in China. Influenza Other Respir Viruses 2020; .

16 Bravi F, Flacco ME, Carradori T, et al. Predictors of severe or lethal COVID-19, including angiotensin converting enzyme inhibitors and angiotensin II receptor blockers, in a sample of infected Italian citizens. PLoS One 2020; 15: e0235248.

17 Koh J, Shah SU, Chua PEY, et al. Epidemiological and clinical characteristics of cases during the early phase of COVID-19 pandemic: a systematic review and meta-analysis. Front Med (Lausanne) 2020; 7: 295.

18 Eddleston J, Lee RU, Doerner AM, et al. Cigarette smoke decreases innate responses of epithelial cells to rhinovirus infection. Am J Respir Cell Mol Biol 2011; 44: 118-126.

19 Alqahtani JS, Oyelade T, Aldhahir AM, et al. Prevalence, severity and mortality associated with COPD and smoking in patients with COVID-19: a rapid systematic review and meta-analysis. PLoS One 2020; 15: e0233147.

20 Cattaruzza MS, Zagà V, Gallus S, et al. Tobacco smoking and COVID-19 pandemic: old and new issues. A summary of the evidence from the scientific literature. Acta Biomed 2020; 91: 106-112. 